\title{
Two channels NIR camera system to detect foreign matter in raw cotton
}

\author{
by S. Böhmer*, H. Budzier*, V. Krause*, G. Gerlach*, T. Pusch** and Ch. \\ Cherif**
}

*Technische Universität Dresden - Institute for Solid State Electronics , Dresden, Germany

${ }^{\star *}$ Technische Universität Dresden - Institute for Textile and Clothing Technology,

Dresden, Germany

\section{Abstract}

Up to now the spinning mills use camera systems in the visible range of light to detect foreign matter like packaging materials in the raw cotton stream during cotton processing. These systems are limited to colored materials since they can not detect white or transparent foils. In this paper a two channels near infrared camera system capable to recognize these types of extraneous substances is presented.

The newly developed camera system is based on two InGaAs line-scan sensors operating in the near infrared range. Both are installed in one single camera head. A $1520 \mathrm{~nm} / 1720 \mathrm{~nm}$ filter pair makes each sensor selective for a specific spectral line within the NIR absorption spectrum that is characteristic for the materials to be detected.

\section{Introduction}

Raw cotton is a material that is irreplaceable for many textile products. Despite of the increasing usage of chemical fibres, its consumption has doubled during the last 40 years. The claim for quality of the products is high. Nevertheless, there is a lot of foreign matter that pollutes the cotton as seen in figures 1 . The International Manufactures Federation classifies 16 different types of polluting substances like tissue, cords, plastic, leaves, feather, sand, metals, oily substances and chemicals. According to the reports of the International Textile Manufacturers Federation (ITMF) of 1995, 1997 and 1999, the degree of pollution is strongly regional-dependant and has grown up to $160 \%$ during the last 10 years. An increase in responsibility of the cotton producers for that problem seems not to be realistic and an aggravation of the situation is expected by the ITMF.
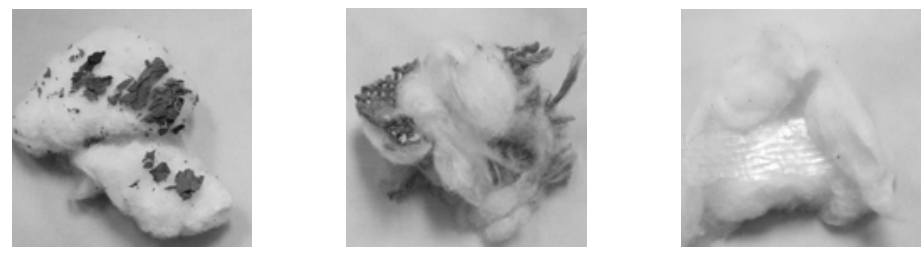

Fig. 1. With different foreign matter polluted raw cotton

As the connecting link between the raw cotton suppliers and the textile industry, the spinning mill is responsible for the damage prevention. They therefore 
need to take care that contamination does not impact negatively the quality of the products. Possible faults in the yarns can lead to costs that exceed the profit and threaten the existence of the companies.

Both the machine-building industry and the manufacturers for test equipment have developed detectors for different machines used in spinning mills. The detection rate varies between $60 \%$ and $80 \%$ in maximum. Those systems are not able to detect white and transparent foils since they show no contrast to cotton in the visible range of light. The detection of these foils would highly improve the quality assurance.

\section{Material Identification using NIR Analysis}

For a qualitative identification of materials it is sufficient to detect specific spectral lines within the near infrared (NIR) absorption band that are characteristic for the materials to be identified. Therefore, a continuous and hence expensive record of the entire spectrum can be avoided. The measurement is reduced to selectively measure the reflection at the characteristic wavelengths. The wavelength selectivity can be achieved using optical filters in front of the sensors.

To identify polypropylene and polyethylene in raw cotton, the spectral range of the NIR is suitable. The absorption bands of oscillations of $\mathrm{CH}-, \mathrm{OH}-$ and $\mathrm{NH}$ - bonds are located in the wavelength range of the NIR $(\lambda=0.75$ to $\lambda=2.5 \mu \mathrm{m})$ making it ideal to identify both polymers and cotton. A second important aspect is that the influence of temperature radiation of the environment is less than in the middle infrared (MIR) range simplifying the measurement arrangement. For the NIR, cost-efficient, fast and sensitive sensor arrays are available that can be run at room temperature. InGaAs diode arrays are especially suitable for fast and un-cooled cameras. They are sensitive at wavelengths between $1000 \mathrm{~nm}$ and 2200nm. Finally, powerful and cost efficient NIR radiation sources are available.

Even though the existence of specific absorption bands are characteristic for a material, the intensity of the bands and the base reflection are dependent on both the measurement conditions like irradiation and detection angle and the concrete sample properties like surface roughness and colouring. It is therefore appropriate to measure the spectrum at peaks in the absorption band to ensure a secure material identification. In addition, only one of the materials to be identified should have a peak at the measured wavelength.

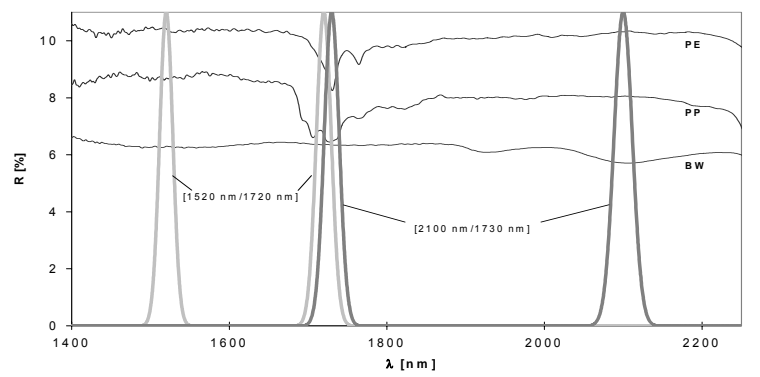

Fig. 2. NIR absorption bands of Polyethylene, Polypropylene and cotton

Figure 2 shows the transmission curves of optical band pass filters together with typical spectrums of polyethylene, polypropylene and cotton. According to earlier studies two spectral channels are adequate to identify these materials. [3] 
Although the $1730 / 2100 \mathrm{~nm}$ wavelength pair has the highest selectivity, the $1520 / 1720$ pair has been chosen. The reason is that the cut-off frequency of standard InGaAs photo diodes is about $1700 \mathrm{~nm}$. The usage of long wave sensors would be disproportional more expensive.

\section{The Measurement System}

A first system has been developed consisting of two NIR line scan cameras which represent the two channels mentioned above. Each camera was equipped with an InGaAs linear array with 256 pixels each operating at $5 \mathrm{kHz}$. The wavelength selectivity was realized using optical filters aligned in front of the sensors. A halogen beamer was used as irradiation source. The system was installed and tested in a test station.

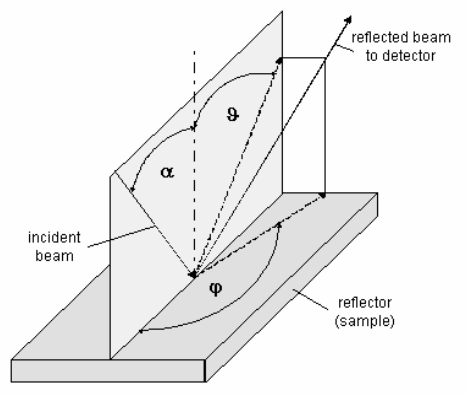

Fig. 3. Detection and Illumination angles

The two cameras were aligned exactly to measure the same spatial point on the surface of a revolving roller. The roller was covered with cotton cords. As test samples, pieces of polyethylene and polypropylene foils were fixed on the cords. The experiments show that the foils can be detected satisfactorily. As shown in figures 4 and 5 , the signals depend on the detection and illumination angles introduced in figure 3 . Since the position of the foils in industrial cotton flows is undefined also, the exact illumination and detection angles will be undefined making the evaluation difficult. Especially the fact that the angles can than be different for the two cameras resulting in different influences on both channels is unfavourable for a secure recognition.

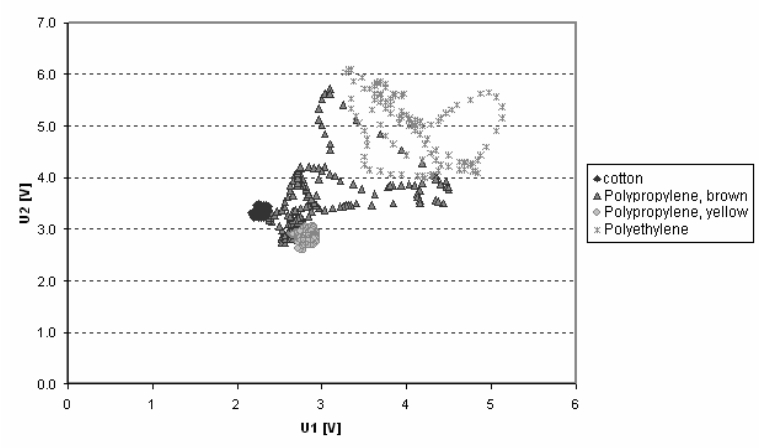

Fig. 4. Clusters of polyethylene, polypropylene and cotton at an illumination angle of $12^{\circ}$ 


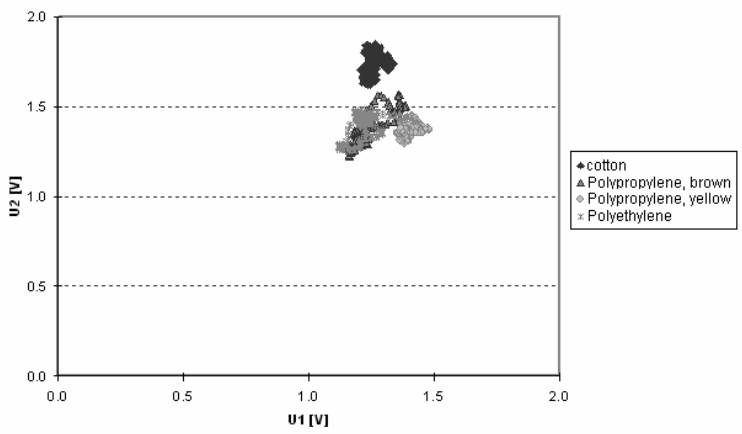

Fig. 5. Clusters of polyethylene, polypropylene and cotton at an illumination angle of $43^{\circ}$

To reduce this aspect the system has been optimized resulting in a completely new camera design. First of all, both sensors were integrated in one camera head allowing exact alignment for exact spatial coverage of the measuring fields. A beam splitter is used to divide the detected radiation into two parts, one for each sensor. The pixel number of 512 for each sensor results in a reduced minimal dimension of the trash that can be detected. The frame rate is again $5 \mathrm{kHz}$.

Figure 6 shows a schematic illustration of the camera head. It basically consists of the optics with beam splitter and the optical filters, a sensor board with the two InGaAs sensors, a power supply, an I/O board to connect the system to the process, and finally a FPGA based embedded SOPC with clock management, image processing and microprocessor. The industrial Camera Link interface provides a high speed data link to transfer the measured data to the PC and a serial communication interface.

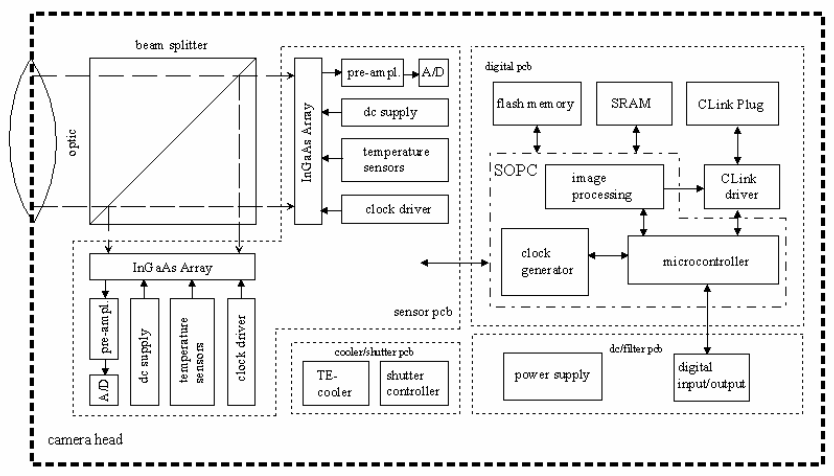

Fig. 6. Functional blocks of the camera head

The interface is used to control the whole system. The microprocessor decodes and executes the received instructions and controls the connected functional blocks. The system can be triggered using the digital I/O board. Two trigger modes are supported: one for edge-triggered and one for gate-triggered operations. The system has a robust housing as seen in figure 7 making it suitable for the use in harsh industrial environments. 


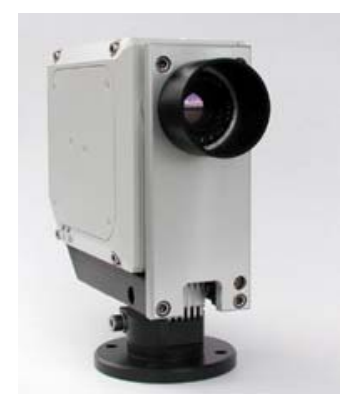

Fig. 7. Camera housing

\section{Signal Processing Algorithms}

The measured signals create a two-dimensional sample space for each pair of corresponding pixels. These two-dimensional sample spaces have to be evaluated. As shown in figure 5 , the different materials create significant clusters within the sample space and can therefore be separated. In general, evaluating the sample space means finding a mapping that separates the clusters. Different methods have been studied starting from classic functions like linear functions or polynomials as well as advanced clustering methods. [3] Even though these methods show good results at the test station, presented in figure 8 , their practical suitability must be doubt. Changes in the process conditions like cotton quality, cotton tint or illumination result in changes in location and size of both the clusters representing the "no-trash" region and the clusters representing the "trash" region within the sample space. The system has to be adapted to those changes by teach-in and learning procedures.

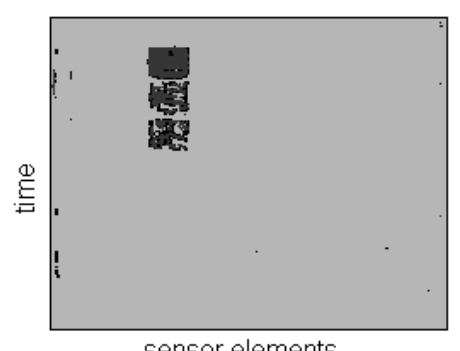

Fig. 8. From the system detected foils

From the practical point of view it would be advantageous to get the system adapting itself to changes of the cluster positions. That means that also the mapping functions have to be self-adjusting. Under this aspect alternative methods have been investigated. Artificial neuronal nets have the property that mapping functions can be found through learning procedures. An analytic description is no longer necessary. A network of connected neurons gets taught with a set of known samples. After the teach-in process the network is able to classify unknown samples. A second advantage is the possibility to get a fuzzy classification allowing the identification of uncertain samples. 
Furthermore, a different self-learning technique has been studied. Selforganizing feature maps can be used to find clusters in a set of unsorted samples. The mapping of the detected cluster positions to the material can be done be teachin or autonomous through statistic evaluation of histograms. The main advantage of this technique is the possibility to track shifts of the clusters with adequate processing intensity.

The suitability of these techniques for usage in the measurement system and under industrial conditions will be investigated when the assembly of the new system is finished.

\section{Application of the system in the spinning mill}

As mentioned in chapter 1 , the machine-building industry has developed a variety of different machines to detect foreign matter in cotton flows. The new camera system will be integrated in a foreign matter extractor in parallel to the existing detector as schematically shown in figure 9 . The cotton flow is fed over a roller that is scanned with the NIR camera. If a foreign substance is detected the air nozzle gets triggered and blows out the polluted cotton. According to the dimensions of the extractor and under consideration of the distance between camera and roller, the system should theoretically be able to detect polyethylene and polypropylene foils with a size larger than $3 \mathrm{~mm} \times 3 \mathrm{~mm}$. The influence of the process conditions and therefore the shifts of the clusters can be investigated under industrial conditions.

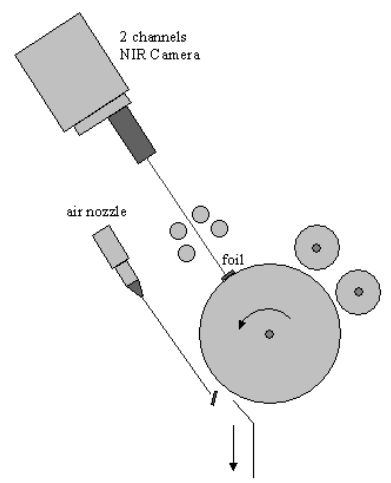

Fig. 9. Foreign substances extractor with NIR camera

\section{Conclusion}

An experimental system to detect foreign matter in cotton flows has been developed. The system was installed at a test station. The suitability of NIR Analysis to recognize foreign substances of polypropylene and polyethylene in cotton has been proven. For industrial usage, the system had to be optimized and a new camera design has been developed with both channels integrated in one camera head making the system robust and easy to handle. Advanced signal processing and clustering methods have been studied to optimize the system for industrial demands. The newly developed system will be integrated in an industrial foreign substances extractor. 


\section{Acknowledgement}

We want to thank the Forschungskuratorium Textil e. V. for financial support of the research project AiF-Nr. 14538 BG. Furthermore, we want to thank those companies which materially and technically support the project, especially Trützschler $\mathrm{GmbH}$, Mönchengladbach for providing the test station as well as DIAS $\mathrm{GmbH}$ Dresden, for the joint camera development.

\section{References}

[1] H. Strolz: ITMF Cotton Contamination Survey 1995. Tagungsband International Cotton Conference (Bremen 1996)

[2] H. Strolz: ITMF Cotton Contamination Survey 1999. Tagungsband International Cotton Conference (Bremen 2000)

[3] B. Vollheim, T. Pusch, H. Budzier, P. Offermann, G. Gerlach: NIR Detection of Contamination in Raw Cotton. World Textile Conference, $2^{\text {nd }}$ AUTEX Conference "Textile Engineering at the dawn of a new millennium: an exciting challenge" (Bruges, Belgium 2002)

[4] T. Pusch, P. Offermann, B. Vollheim, H. Budzier, V. Krause, G. Gerlach: Fremdstofferkennung in Rohbaumwolle, Melliand Textilberichte 83 (2002) H. 4, S. $216-218$ 